\title{
Composição florística do componente arbustivo-arbóreo em dois trechos de floresta estacional semidecidual na Mata do Paraíso, Viçosa, MG
}

\author{
Sheila Isabel do Carmo Pinto \\ Sebastião Venâncio Martins² \\ Bruno da Silva Moretti3
}

\section{Resumo}

É grande a demanda por estudos ecológicos em florestas que possam embasar trabalhos de recuperação e conservação da biodiversidade. Entre esses estudos, primordialmente está o levantamento da flora. Nesse sentido, realizou-se o estudo das variações florísticas do componente arbustivo-arbóreo em dois trechos de Floresta Estacional Semidecidual, denominados neste estudo de floresta inicial e floresta madura, situados na Reserva Florestal Mata do Paraíso, em Viçosa, Minas Gerais. As espécies arbóreo-arbustivas foram amostradas dentro de 20 parcelas de $10 \times 30$ m, 10 parcelas em cada trecho de floresta, sendo considerados apenas os indivíduos com diâmetro a 1,30 m do solo $(\mathrm{DAP}) \geq 4,8 \mathrm{~cm}$. Na floresta inicial foram amostradas 55 espécies pertencentes a 47 gêneros e 27 famílias. A família com maior riqueza florística foi Fabaceae, com 10 espécies (18\%), seguida de Euphorbiaceae (cinco espécies; 9\%), Annonaceae, Lauraceae, Meliaceae e Salicaceae (três espécies; 6\%). Na floresta madura foram amostradas 78 espécies distribuídas em 62 gêneros e 31 famílias. A família com maior riqueza florística foi Fabaceae, com 13 espécies (17\%), seguida de Lauraceae e Myrtaceae (seis espécies; 8\%), Euphorbiaceae, Sapindaceae e Salicaceae (cinco espécies; 6\%), Annonaceae e Meliaceae (quatro espécies; 5\%), Rubiaceae e Moraceae (três espécies; 4\%). 0 grupo ecológico que mais se destacou nos dois trechos de floresta foi o das secundárias iniciais, seguido pelas secundárias tardias na floresta madura e pelas pioneiras na floresta inicial. A variabilidade na composição florística e na proporção de espécies em cada grupo ecológico foi resultante das variações na intensidade da ação antrópica nestas florestas e do tempo de regeneração florestal.

Palavras-chaves: Sucessão ecológica. Levantamento florístico. Grupos ecológicos.

\section{Introdução}

Nas últimas décadas, houve considerável avanço nos estudos de comunidades florestais, principalmente por causa de sua importância para a conservação da diversidade biológica. Essa importância se torna cada dia mais acentuada devido ao processo desordenado de ocupação, que nas mais diversas regiões do país, tem transformado formações florestais contínuas em fragmentos (RODRIGUES et al., 2003; OLIVEIRA FILHO et al., 1994). 
Na Zona da Mata de Minas Gerais, o processo de fragmentação e degradação da vegetação florestal nativa não foi diferente do que ocorreu em todo o Sudeste Brasileiro, restando atualmente fragmentos florestais isolados nos topos de morros de difícil acesso, em variados estádios de sucessão secundária.

Desta forma, a avaliação da diversidade biológica contida nos fragmentos florestais constitui ferramenta importante para a avaliação dos potenciais de perdas e conservação dos recursos naturais em longo prazo, uma vez que permite compreender a organização espacial da comunidade e a direção das mudanças nos processos ecológicos (ESPÍRITO SANTO et al., 2002).

Assim, estudos sobre a composição florística e a ecologia das comunidades vegetais são fundamentais para embasar quaisquer iniciativas de preservação e conservação de remanescentes florestais (OLIVEIRA FILHO et al., 1994), bem como para orientar medidas de manejo a serem adotadas, a partir do entendimento do processo da dinâmica sucessional instaurada nos diferentes estádios sucessionais de uma comunidade florestal (BRAGA et al., 2011).

O presente trabalho teve por objetivo descrever a composição de espécies do componente arbóreo-arbustivo, bem como a distribuição destas espécies em diferentes grupos ecológicos, em dois trechos de Floresta Estacional Semidecidual em estádios sucessionais distintos na Reserva Florestal Mata do Paraíso, em Viçosa, MG.

\section{Material e métodos}

O estudo foi conduzido na Reserva Florestal Mata do Paraíso (2048'07"S e 4251'31"W), pertencente à Universidade Federal de Viçosa (UFV), que possui 195 ha e altitude variando de 690 a 800 m, está localizada no município de Viçosa, Zona da Mata de Minas Gerais (BRAZ et al., 2002).

O clima na região é do tipo Cwb (Köppen), mesotérmico com verões quentes e chuvosos e invernos frios e secos. A temperatura média anual é de $21,8^{\circ} \mathrm{C}$ e a precipitação pluviométrica média anual de 1.314,2 mm (CASTRO et al., 1983). A vegetação da reserva é composta por trechos de Floresta Estacional Semidecidual (VELOSO et al., 1991).

Os trechos de floresta escolhidos para este estudo apresentam diferentes históricos de perturbação e regeneração que foram caracterizados por Silva Júnior et al. (2004). Um desses trechos, denominado neste estudo de floresta inicial, encontra-se em processo de regeneração florestal em pastagem de Melinis minutiflora P. Beauv. desde 1963. O outro trecho de floresta, denominado floresta madura, sofreu somente exploração seletiva de madeira, constituindo um núcleo de floresta bem conservado, livre de distúrbios antrópicos nas últimas cinco décadas.

O levantamento florístico foi realizado em 10 parcelas permanentes de $10 \times 30 \mathrm{~m}$, alocadas com intervalos de $10 \mathrm{~m}$ entre parcelas, em cada trecho de floresta, totalizando uma área amostral de 0,6 ha. Todos os indivíduos com diâmetro a $1,30 \mathrm{~m}$ do solo (DAP) $\geq 4,8 \mathrm{~cm}$ foram identificados. 0 material botânico foi identificado por meio de consultas ao herbário VIC da Universidade Federal de Viçosa (UFV) e as espécies foram classificadas em famílias de acordo com o sistema do Angiosperm Phylogeny Group II (APG II, 2003).

As espécies arbustivo-arbóreas amostradas foram classificadas em categorias sucessionais seguindo os critérios adotados por Gandolfi et al. (1995), além de classificações realizadas em outros estudos (MARTINS, 1999; MARTINS et al., 2002; 2004; PAULA et al., 2002; PEIXOTO et al., 2004; SILVA et al., 2003). As espécies foram classificadas em pioneiras (espécies dependentes de luz que não ocorrem no sub-bosque, desenvolvendo-se em clareiras ou nas bordas da floresta); secundárias iniciais (espécies que ocorrem em condições de sombreamento médio ou luminosidade não muito intensa, em clareiras pequenas, bordas de clareiras grandes, bordas da floresta ou no sub-bosque pouco sombreado); secundárias tardias (espécies que se desenvolvem no sub-bosque sob 
sombra leve ou densa, podendo permanecer neste ambiente por toda a vida ou crescer e alcançar o dossel ou tornar-se emergente) e sem caracterização (espécies que por falta de informações não puderam ser incluídas em nenhuma das categorias anteriores).

Para comparar floristicamente os trechos de floresta estudados foi elaborada uma matriz de presença e ausência de espécies e a partir desta construído um dendrograma de similaridade entre todas as parcelas da floresta inicial e floresta madura, com todas as espécies amostradas, por meio do programa FITOPAC 1 (SHEPHERD, 1996). Na elaboração do dendrograma foram utilizados o índice de similaridade de Jaccard (PIELOU, 1975) e o método de agrupamento pela média de grupo (UPGMA).

\section{Resultados e discussão}

Ao todo, foram amostradas 100 espécies no levantamento fitossociológico nos dois trechos de floresta, sendo 33 espécies comuns às duas florestas (Tabela 1). Na floresta inicial foi encontrado um total de 55 espécies. A família com maior riqueza florística foi Fabaceae, com 10 espécies (18\%), sendo cinco espécies pertencentes à subfamília Caesalpinioideae, duas espécies à subfamília Faboideae e três espécies à subfamília Mimosoideae, seguida da família Euphorbiaceae (cinco espécies; 9\%), Annonaceae, Lauraceae, Meliaceae e Salicaceae (três espécies; 6\%). Estas famílias representam $49 \%$ da flora amostrada, podendo-se ainda observar que $25 \%$ das famílias foram amostradas por meio de uma única espécie (Tabela 1 ).

Tabela 1. Lista das espécies arbustivo-arbóreas amostradas nas florestas inicial e madura, na Reserva Florestal Mata do Paraíso, em Viçosa - MG, dispostas em ordem alfabética de famílias.

\begin{tabular}{cccc}
\hline Famílias/ Espécies & \multicolumn{2}{c}{ Floresta } & CS \\
\cline { 2 - 3 } ANNONACEAE & Inicial & Madura & \\
Annonaceae sp. & & $\mathrm{X}$ & $\mathrm{SC}$ \\
Annona cacans Warm. & $\mathrm{X}$ & $\mathrm{SI}$ \\
$\quad$ Guatteria sp. & $\mathrm{X}$ & $\mathrm{X}$ & $\mathrm{SC}$ \\
Guatteria nigrescens Mart. & $\mathrm{X}$ & $\mathrm{ST}$ \\
Xylopia sericea A St.-Hil. & & $\mathrm{X}$ & $\mathrm{SI}$ \\
Rollinia sylvatica (A St.-Hil.) Martius & & \\
APOCYNACEAE & & $\mathrm{X}$ & $\mathrm{ST}$ \\
Himatanthus phagedaenicus (Mart.) Woodson & & \\
ARALIACEAE & $\mathrm{X}$ & $\mathrm{SI}$ \\
Schefflera morototoni (Aubl.) Maguire & & $\mathrm{ST}$ \\
ARECACEAE & $\mathrm{X}$ & $\mathrm{SI}$ \\
Euterpe edulis Mart. & & & $\mathrm{PI}$ \\
Syagrus romanzoffiana (Cham.) Glassman & $\mathrm{X}$ & $\mathrm{PI}$
\end{tabular}




\begin{tabular}{|c|c|c|c|}
\hline \multicolumn{4}{|l|}{ BIGNONIACEAE } \\
\hline Jacaranda macrantha Cham. & $x$ & $x$ & $\mathrm{Pl}$ \\
\hline Sparattosperma leucanthum (Vell.) K. Schum. & $X$ & & $\mathrm{PI}$ \\
\hline \multicolumn{4}{|l|}{ BURSERACEAE } \\
\hline Protium heptaphyllum (Aubl.) Marchand & $X$ & $x$ & $\mathrm{SI}$ \\
\hline Protium warmingiana March, L. & $x$ & $x$ & ST \\
\hline \multicolumn{4}{|l|}{ CLUSIACEAE } \\
\hline Garcinia gardneriana (Planch. \& Triana) Zappi & & $x$ & ST \\
\hline Tovomitopsis saldanhae Engl. & & $x$ & ST \\
\hline Vismia guianensis (Aubl.) Pers. & $x$ & & $\mathrm{Pl}$ \\
\hline \multicolumn{4}{|l|}{ ERYTHROXYLACEAE } \\
\hline Erythroxylum pelleterianum A St.-Hil. & $X$ & & $\mathrm{SI}$ \\
\hline \multicolumn{4}{|l|}{ EUPHORBIACEAE } \\
\hline Alchornea glandulosa Poepp. & $x$ & $x$ & SI \\
\hline Croton floribundus Spreng. & $x$ & $x$ & $\mathrm{PI}$ \\
\hline Hyeronima alchorneoides Allemão & $x$ & $x$ & $\mathrm{SI}$ \\
\hline Manihot pilosa Pohl & $X$ & $x$ & $\mathrm{PI}$ \\
\hline Sapium glandulatum (Vell.) Pax & $x$ & $x$ & $\mathrm{PI}$ \\
\hline \multicolumn{4}{|l|}{ FABACEAE } \\
\hline Anadenanthera macrocarpa (Benth.) Brenan & $X$ & $x$ & $\mathrm{SI}$ \\
\hline Apuleia leiocarpa (Vogel) J. F. Macbr. & $x$ & $x$ & SI \\
\hline Bauhinia forficata Link & $x$ & $\mathrm{X}$ & $\mathrm{SI}$ \\
\hline Dalbergia nigra Allemão ex Benth. & $x$ & $x$ & $\mathrm{SI}$ \\
\hline Inga edulis Mart. & & $X$ & $\mathrm{SI}$ \\
\hline Inga marginata Willd. & & $X$ & $\mathrm{SI}$ \\
\hline Lonchocarpus muehlbergianus Hassl. & & $x$ & SI \\
\hline Machaerium nyctitans (Vells.) Benth. & $X$ & $X$ & $\mathrm{SI}$ \\
\hline Peltophorum dubium (Spreng.) Taub. & $x$ & & $\mathrm{SI}$ \\
\hline Piptadenia gonoacantha (Mart.) J. F. Macbr. & $x$ & $\mathrm{X}$ & $\mathrm{SI}$ \\
\hline Platycyamus regnellii Benth. & & $x$ & ST \\
\hline Pseudopiptadenia contorta (DC.) & & $X$ & $\mathrm{SI}$ \\
\hline Senna macranthera (Vell.) Irwin \& Barneby & $x$ & & $\mathrm{PI}$ \\
\hline Senna multijuga (Rich.) H. S. Irwin \& Barneby & $X$ & $x$ & $\mathrm{SI}$ \\
\hline Stryphnodendron polyphyllum Mart. & $X$ & & $\mathrm{SI}$ \\
\hline Swartzia myrtifolia Sm. & & $X$ & ST \\
\hline \multicolumn{4}{|l|}{ ICACINACEAE } \\
\hline Citronella paniculata (Mart.) R. A Howard & & $X$ & ST \\
\hline \multicolumn{4}{|l|}{ LACISTEMATACEAE } \\
\hline Lacistema pubescens Mart. & $X$ & $x$ & $\mathrm{SI}$ \\
\hline \multicolumn{4}{|l|}{ LAURACEAE } \\
\hline Endlicheria paniculata (Spreng.) J. F. Macbr. & & $x$ & $\mathrm{SI}$ \\
\hline Nectandra sp. & & $x$ & SC \\
\hline
\end{tabular}




\begin{tabular}{|c|c|c|c|}
\hline Nectandra lanceolata Nees & $x$ & $x$ & ST \\
\hline Nectandra oppositifolia Nees & $x$ & $x$ & ST \\
\hline Ocotea corymbosa (Meisn.) Mez, & $x$ & $x$ & $\mathrm{SI}$ \\
\hline Ocotea odorifera (Vellozo) Rohwer & & $x$ & ST \\
\hline \multicolumn{4}{|l|}{ LECYTHIDACEAE } \\
\hline Cariniana estrellensis (Raddi) Kuntze & & $x$ & ST \\
\hline \multicolumn{4}{|l|}{ MALVACEAE } \\
\hline Luehea grandiflora Mart. & $x$ & & $\mathrm{SI}$ \\
\hline Pseudobombax grandiflorum (Cav.) A Robyns & & $x$ & $\mathrm{SI}$ \\
\hline \multicolumn{4}{|l|}{ MELASTOMATACEAE } \\
\hline Miconia cinnamomifolia (DC.) Naudin & $x$ & & $\mathrm{Pl}$ \\
\hline Miconia pusilliflora Beurl. & & $x$ & SC \\
\hline \multicolumn{4}{|l|}{ MELIACEAE } \\
\hline Cabralea canjerana (Vell.) Mart. & $x$ & $x$ & $\mathrm{SI}$ \\
\hline Guarea macrophylla Vahl & & $X$ & ST \\
\hline Trichilia catigua A Juss. & & $X$ & $\mathrm{SI}$ \\
\hline Trichilia lepidota Mart. & $x$ & $X$ & ST \\
\hline Trichilia pallida Sw. & $x$ & & $\mathrm{SI}$ \\
\hline \multicolumn{4}{|l|}{ MORACEAE } \\
\hline Acanthinophyllum ilicifolium (Spreng.) Burger & & $X$ & $\mathrm{SI}$ \\
\hline Ficus insipida Willd. & & $X$ & $\mathrm{SI}$ \\
\hline Sorocea bonplandii (Baill.) W. C. Burger & $x$ & $X$ & ST \\
\hline \multicolumn{4}{|l|}{ MYRISTICACEAE } \\
\hline Virola oleifera (Schott) A C. Sm. & & $X$ & $\mathrm{SI}$ \\
\hline \multicolumn{4}{|l|}{ MYRTACEAE } \\
\hline Eugenia speciosa Cambess. & & $X$ & ST \\
\hline Myrcia sp. & & $X$ & SC \\
\hline Myrciaria cauliflora (Mart.) O. Berg & & $X$ & ST \\
\hline Myrcia fallax (Rich.) DC. & $\mathrm{X}$ & $X$ & $\mathrm{SI}$ \\
\hline Myrcia rostrata DC. & $\mathrm{X}$ & $X$ & $\mathrm{Pl}$ \\
\hline Myrcia sphaerocarpa DC. & & $X$ & ST \\
\hline \multicolumn{4}{|l|}{ NYCTAGINACEAE } \\
\hline Guapira opposita (Vell.) Reitz & $x$ & $X$ & $\mathrm{SI}$ \\
\hline \multicolumn{4}{|l|}{ PHYTOLACACEAE } \\
\hline Seguieria langsdorffii Moq. & & $x$ & $\mathrm{SI}$ \\
\hline \multicolumn{4}{|l|}{ PICRAMNACEAE } \\
\hline Picramnia regnelli Engl. & & $x$ & ST \\
\hline \multicolumn{4}{|l|}{ RHAMNACEAE } \\
\hline Colubrina glandulosa Perkins & $x$ & & $\mathrm{SI}$ \\
\hline ROSACEAE & & & \\
\hline
\end{tabular}




\begin{tabular}{|c|c|c|c|}
\hline Prunus sellowii Koehne & $x$ & $x$ & $\mathrm{SI}$ \\
\hline \multicolumn{4}{|l|}{ RUBIACEAE } \\
\hline Amaioua guianensis Aubl. & & $x$ & ST \\
\hline Coffea arabica L. & & $x$ & SC \\
\hline Psychotria carthaginensis Jacq. & & $x$ & ST \\
\hline Psychotria vellosiana Vell. & $x$ & & ST \\
\hline \multicolumn{4}{|l|}{ RUTACEAE } \\
\hline Dictyoloma vandellianum A H. L. Juss & $x$ & & $\mathrm{SI}$ \\
\hline Zanthoxylum rhoifolium Lam. & $x$ & $x$ & $\mathrm{Pl}$ \\
\hline \multicolumn{4}{|l|}{ SALICACEAE } \\
\hline Carpotroche brasiliensis (Raddi) Endl. & $X$ & $X$ & ST \\
\hline Casearia arborea (Rich.) Urb. & & $x$ & $\mathrm{SI}$ \\
\hline Casearia gossypiosperma Briq. & $x$ & $x$ & SI \\
\hline Casearia sylvestris Sw. & $x$ & $x$ & $\mathrm{PI}$ \\
\hline Xylosma salzmannii (Clos) Eichler & & $x$ & SI \\
\hline \multicolumn{4}{|l|}{ SAPINDACEAE } \\
\hline Allophylus edulis (A St.-Hil., Cambess. \& A Juss) & & $x$ & $\mathrm{Pl}$ \\
\hline Allophylus sericeus Radlk. & & $x$ & SI \\
\hline Cupania vernalis Cambess. & & $x$ & SI \\
\hline Matayba elaeagnoides Radlk. & $x$ & $x$ & SI \\
\hline Matayba leocodictya Radlk. & & $x$ & ST \\
\hline \multicolumn{4}{|l|}{ SAPOTACEAE } \\
\hline Chrysophyllum flexuosum Mart. & & $x$ & ST \\
\hline \multicolumn{4}{|l|}{ SIPARUNACEAE } \\
\hline Siparuna arianae V. Pereira & & $x$ & ST \\
\hline Siparuna guianensis Aubl. & $x$ & & ST \\
\hline \multicolumn{4}{|l|}{ SOLANACEAE } \\
\hline Cestrum sp. & & $x$ & $\mathrm{SC}$ \\
\hline Solanum sp. & & $x$ & SC \\
\hline Solanum cernnum Vell. & $x$ & & $\mathrm{PI}$ \\
\hline Solanum leucodendron Sendtn. & $x$ & & $\mathrm{PI}$ \\
\hline \multicolumn{4}{|l|}{ URTICACEAE } \\
\hline Cecropia glaziovi Snethlage & $x$ & & $\mathrm{PI}$ \\
\hline Cecropia hololeuca Miq. & $x$ & & $\mathrm{PI}$ \\
\hline \multicolumn{4}{|l|}{ VOCHYSIACEAE } \\
\hline Vochysiaceae sp. & & $x$ & SC \\
\hline
\end{tabular}

$\mathrm{CS}$ = categoria sucessional $(\mathrm{PI}=$ espécies pioneiras, $\mathrm{SI}=$ espécies secundárias iniciais, $\mathrm{ST}$ = espécies secundárias tardias e SC $=$ sem caracterização).

Fonte: Elaboração própria. 
A floresta madura apresentou 78 espécies. De forma análoga à floresta inicial, a família com maior riqueza na floresta madura foi a Fabaceae, com 13 espécies (17\%), sendo três espécies pertencentes à subfamília Caesalpinioideae, cinco espécies à subfamília Faboideae e cinco espécies à subfamília Mimosoideae (Figura 2), seguida de Lauraceae e Myrtaceae (seis espécies; 8\%), Euphorbiaceae, Sapindaceae e Salicaceae (cinco espécies; 6\%), Annonaceae e Meliaceae (quatro espécies, $5 \%$ ), Moraceae e Rubiaceae (três espécies; $4 \%$ ), totalizando $69 \%$ da flora amostrada. As famílias amostradas por uma única espécie totalizaram 22\% (Tabela 1).

O número total de espécies amostradas neste estudo (100) está de acordo com levantamentos efetuados em Florestas Estacionais Semideciduais na região de Viçosa, Minas Gerais. Valores inferiores foram citados por Silva et al. (2000), 91 espécies, e por Paula et al. (2002), 94 espécies. Valores superiores foram obtidos por Ribas et al. (2003), 107 espécies, e por Silva et al. (2004), 124 espécies.

Estudos indicam uma tendência de aumento no número de espécies ao longo do processo de sucessão secundária em florestas tropicais (TABARELLI et al., 1994; TURNER et al., 1997; AIDAR et al., 2001; RODRIGUES et al., 2004), que pode ser atribuída ao aumento na partição de recursos por maior número de espécies devido à melhoria na ciclagem de nutrientes, fertilidade do solo e sombreamento, seguindo um modelo sucessional de facilitação (CONNEL; SLATYER, 1977).

Consultas a trabalhos desenvolvidos na região de Viçosa mostram que, nos levantamentos florísticos de Florestas Estacionais Semideciduais, as famílias Fabaceae, Euphorbiaceae, Lauraceae, Myrtaceae, Rubiaceae e Annonaceae têm assumido grande importância (PAULA et al., 2002; RIBAS et al., 2003; MARANGON et al., 2003; SILVA et al., 2003; SILVA et al., 2004). Essas famílias foram citadas por Lopes et al. (2002) como as famílias de maior riqueza específica nos remanescentes de floresta estacional semidecídua da Zona da Mata Mineira, após estudo comparativo da composição florística de seis fragmentos da região. A grande importância dessas famílias nas florestas estacionais já havia sido ressaltada por Leitão-Filho (1982). A ocorrência das mesmas famílias com elevada riqueza específica nos remanescentes de floresta estacional semidecídua na porção leste de Minas Gerais demonstra a considerável ligação florística entre as áreas, condicionada pela similaridade de condições ambientais compartilhadas (FERREIRA JÚNIOR et al., 2007).

Na floresta inicial, os gêneros que apresentaram maior riqueza florística foram Protium, Senna, Nectandra, Trichilia, Myrcia, Casearia, Solanum e Cecropia (duas espécies). Os demais gêneros (39) são representados por uma única espécie, o que equivale a $83 \%$ dos gêneros amostrados. Na floresta madura, os gêneros com maior riqueza florística foram Myrcia (quatro espécies), Nectandra e Casearia (três espécies), Protium, Inga, Ocotea, Guatteria, Trichilia, Allophylus e Matayba (duas espécies). Os demais gêneros (54) são representados por apenas uma espécie, correspondendo a $84 \%$ dos gêneros amostrados.

Das 100 espécies amostradas neste estudo, cinco encontram-se na lista da flora ameaçada de extinção do Estado de Minas Gerais (MENDONÇA; LINS, 2000). Euterpe edulis, Ocotea odorifera e Dalbergia nigra estão inseridas na lista oficial das espécies da flora brasileira ameaçadas de extinção segundo a Instrução Normativa Nº 06, do Ministério do Meio Ambiente, de 23 de setembro de 2008. Vernonanthura diffusa e Tovomtopsis saldanhae são incluídas na lista de espécies presumivelmente ameaçadas de extinção da flora de Minas Gerais. Este fato ressalta ainda mais a importância da preservação deste fragmento, não só pela expressiva dimensão do fragmento florestal, mas por constituir área propícia à manutenção de populações de espécies sujeitas à extinção, principalmente, Euterpe edulis (Tabela 2), muito atrativa à avifauna e pouco frequente nos demais fragmentos da região.

A classificação sucessional das espécies (Tabela 2) revelou que, em relação ao número de espécies por categoria sucessional, as secundárias iniciais ocuparam a primeira posição nos dois trechos de floresta estudados, seguidas das pioneiras na floresta inicial e das secundárias tardias na floresta madura. Quanto ao número de indivíduos amostrados por categoria sucessional, as pioneiras sobressaíram na floresta inicial e as secundárias tardias na floresta madura. 
Tabele 2. Categorias sucessionais das espécies arbóreo-arbustivas amostradas nas florestas inicial e madura na Reserva Florestal Mata do Paraíso em Viçosa, MG, Brasil.

\begin{tabular}{ccccccccc}
\hline & \multicolumn{4}{c}{ Floresta inicial } & \multicolumn{3}{c}{ Floresta madura } \\
\cline { 2 - 9 } Categoria sucessional & \multicolumn{2}{c}{ Espécies } & \multicolumn{2}{c}{ Indivíduos } & \multicolumn{2}{c}{ Espécies } & \multicolumn{2}{c}{ Indivíduos } \\
\cline { 2 - 9 } & $N^{0}$ & $\%$ & $N^{0}$ & $\%$ & $N^{\circ}$ & $\%$ & $N^{0}$ & $\%$ \\
\hline Pioneiras & 17 & 30,9 & 175 & 43,9 & 9 & 11,5 & 31 & 5,0 \\
Secundárias Iniciais & 29 & 52,7 & 156 & 39,1 & 33 & 42,3 & 231 & 37,1 \\
Secundárias Tardias & 9 & 16,4 & 68 & 17,0 & 27 & 34,7 & 347 & 55,7 \\
Sem caracterização & 0 & 0 & 0 & 0 & 9 & 11,5 & 14 & 2,2 \\
\hline Total & 55 & 100,0 & 399 & 100,0 & 78 & 100,0 & 623 & 100,0 \\
\hline
\end{tabular}

Fonte: Elaboração própria

Na floresta inicial, as famílias Asteraceae, Bignoniaceae, Clusiaceae, Melastomataceae, Solanaceae e Urticaceae foram representadas exclusivamente por espécies pioneiras, enquanto a família Euphorbiaceae foi predominantemente representada por pioneiras. As famílias Annonaceae, Araliaceae, Arecaceae, Erythroxylaceae, Fabaceae, Lacistemataceae, Malvaceae, Meliaceae, Nyctaginaceae, Rhamnaceae, Rosaceae e Sapindaceae foram predominantemente representadas por secundárias iniciais. As famílias Lauraceae, Moraceae, Rubiaceae e Siparunaceae foram compostas predominantemente por espécies classificadas como secundárias tardias.

Analisando o caráter sucessional de espécies arbóreo-arbustivas em uma floresta semidecídua de Guarulhos, SP, Gandolfi et al. (1995) também encontraram famílias exclusivamente pioneiras (Urticaceae e Clusiaceae), secundárias iniciais (Burseraceae e Nyctaginaceae) e secundárias tardias (Erythroxylaceae, Lecythidaceae e Sapotaceae), além de famílias importantes como Euphorbiaceae e Fabaceae, em que não se observou uma exclusividade ou predominância de caráter sucessional.

$\mathrm{Na}$ floresta madura as famílias Asteraceae, Bignoniaceae, Euphorbiaceae e Rutaceae foram predominantemente representadas por espécies pioneiras. As famílias Fabaceae, Lacistemataceae, Malvaceae, Moraceae, Myristicaceae, Nyctaginaceae, Phytolacaceae, Rosaceae, Salicaceae e Sapindaceae foram predominantemente representadas por espécies secundárias iniciais. As famílias Apocynaceae, Arecaceae, Clusiaceae, Icacinaceae, Lauraceae, Lecythidaceae, Picramnaceae, Rubiaceae, Sapotaceae e Siparunaceae foram compostas predominantemente por espécies classificadas na categoria de secundárias tardias.

As espécies classificadas como secundárias tardias e pioneiras contribuíram com baixos percentuais tanto de espécies como de indivíduos para as florestas inicial e madura, respectivamente. Estes resultados indicam que a diversidade de espécies encontrada na floresta inicial é representada principalmente por espécies iniciais na sucessão secundária, enquanto na floresta madura, estas são representadas por espécies tardias.

Os resultados evidenciam que, apesar da predominância de espécies pioneiras e secundárias iniciais na floresta inicial, um certo nível de sombreamento proporcionado pelo dossel já possibilita a ocorrência de espécies tolerantes à sombra, típicas de sub-bosque, o que reflete o avanço sucessional também neste trecho de floresta. Portanto, pode-se deduzir que com o passar do tempo as diferenças florísticas entre os dois trechos tendem a ser minimizadas.

Assim, tendo como parâmetro a Resolução Conama n 10, de $1^{\circ}$ de outubro de 1993 (publicada no DOU n² 209, de 3 de novembro de 1993, Seção 1, páginas 16497-16498), a floresta inicial encontra-se em fase de transição, do estádio inicial para o médio, enquanto o estádio de sucessão da floresta madura se enquadra como o de floresta avançada, conforme os parâmetros básicos descritos para a vegetação de Mata Atlântica.

As relações florísticas visualizadas por meio do dendrograma gerado pela análise de agrupamento utilizando o método da média de grupo (UPGMA), com base no índice de similaridade de 
Jaccard, são representadas na Figura 1. O dendrograma mostra, graficamente, que foram formados dois grandes grupos de parcelas, ficando evidente que estes dois grupos apresentam uma similaridade em torno de $10 \%$. Como duas comunidades são consideradas floristicamente diferentes quando o valor do índice de Jaccard entre elas for igual ou superior a 25\% (MUELLER-DOMBOIS; ELLENBERG, 1974), ao nível de 17\% ocorreu a separação da parcela 10 das demais parcelas do grupo 1 (floresta inicial) e ao nível de $23 \%$ ocorreu a separação da parcela 20 das demais parcelas do grupo 2 (floresta madura).

FIGURA 1. Dendrograma obtido pelo método da média de grupo (UPGMA), com base no índice de Jaccard, para os dados de presença e ausência das espécies das florestas inicial (parcelas de 1 a 10) e madura (parcelas de 11 a 20), na Reserva Florestal Mata do Paraíso, Viçosa, MG.

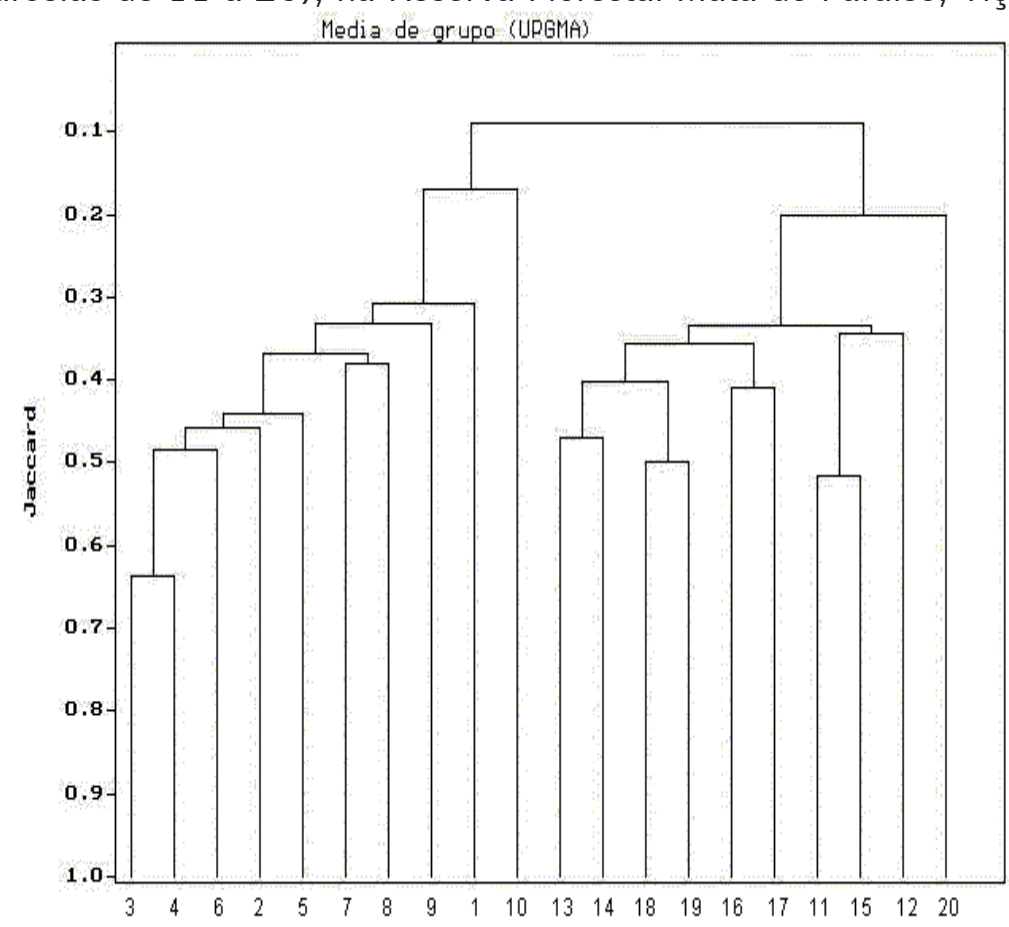

Fonte: Elaboração própria

Os mais altos índices de similaridade foram obtidos entre as parcelas pertencentes ao mesmo trecho de floresta. Esta maior semelhança está relacionada à proximidade espacial, o que implica em históricos de perturbação e regeneração semelhantes, resultando em composições florísticas similares e mesmo estádio de sucessão secundária.

Segundo Muller Dumbois e Ellenberg (1974), os remanescentes de floresta podem ser considerados floristicamente semelhantes sempre que apresentarem pelo menos $25 \%$ de similaridade. Neste contexto, as florestas inicial e madura podem ser consideradas pouco similares entre si. A heterogeneidade entre os dois trechos de floresta tão próximos pode decorrer de variações de condições ambientais, das preferências ecológicas das espécies e também dos diversos graus de perturbação provocados pelo homem (BERTONI; MARTINS, 1987).

A baixa similaridade florística entre as florestas inicial e madura, com somente 33 espécies comuns às duas áreas, faz com que a floresta inicial tenha todas suas parcelas formando um grupo inequívoco, divergindo de todas as parcelas da floresta madura, que formaram outro grupo distinto na análise de agrupamento. Esses grupos de similaridade assim estabelecidos estão relacionados às condições ecológicas destas comunidades e corroboram a heterogeneidade florística entre as florestas 
inicial e madura, sendo, as variáveis ambientais e intervenções antrópicas responsáveis, no decorrer do processo de sucessão, pela manutenção da diversidade florística da Floresta Estacional Semidecidual.

\title{
4 Conclusão
}

A variabilidade na composição florística das florestas inicial e madura, bem como na proporção de espécies em cada grupo ecológico, foi determinada pelas variações na intensidade da ação antrópica sofrida e pelo tempo de regeneração florestal de cada fragmento florestal.

\section{Floristic composition of woody component in two stretches of semideciduous forest in Mata do Paraíso, Viçosa, MG}

\begin{abstract}
It is great the demand for ecological studies in forests that can support recovery efforts and biodiversity conservation. Flora survey is primarily among these studies. Accordingly, there was the study of the floristic variation of woody component in two stretches of semideciduous forest, called in this study initial forest and mature forest, located in the Forest Reserve Mata do Paraíso, in Viçosa, Minas Gerais. The tree species were sampled in 20 plots of $10 \times 30 \mathrm{~m}, 10$ plots in each forest stand, $\mathrm{s}$ being considered only individuals with a diameter at $1.30 \mathrm{~m}$ (DAP) $4.8 \mathrm{~cm}$. In the initial forest were sampled 55 species belonging to 47 genera and 27 families. The family with the highest richness of species were Fabaceae with 10 species (18\%), followed by Euphorbiaceae (five species; 9\%), Annonaceae, Lauraceae, Meliaceae and Salicaceae (three species; $6 \%$ ). In the mature forest were sampled 78 species in 62 genera and 31 families. The family with the highest richness of species were Fabaceae with 13 species (17\%), followed by Lauraceae and Myrtaceae (six species; 8\%), Euphorbiaceae, Sapindaceae and Salicaceae (five species; 6\%), Annonaceae and Meliaceae (four species; 5\%), Rubiaceae and Moraceae (three species; 4\%). The ecological group that stood out in two sections of the secondary forest was the initial one, followed by late successional forest and mature forest in the pioneering stages. The variability in floristic composition and proportion of species in each ecological group was resulting from variations in the intensity of human activities in these forests and forest regeneration time.
\end{abstract}

Keywords: Ecological succession. Floristic survey. Ecological groups.

\section{Referências bibliográficas}

AIDAR, M. P. M.; GODOY, J.R.L.; BERGMANN, J.; JOLY, C.A. Atlantic Forest succession over calcareous soil Parque Estadual Turístico do Alto da Ribeira - PETAR, SP. Revista Brasileira de Botânica, São Paulo, v. 24, no 4, p. 455-469, 2001. Disponível em: <http://www.scielo.br/scielo. php?script $=$ sci_arttext\&pid $=$ S0100-84042001000400012\&nrm $=i s o \& t$ lng $=p t>$. Acesso em: 13 maio 2013 
ANGIOSPERM PHYLOGENY GROUP II. An update of the Angiosperm Phylogeny Group classification for the orders and families of flowering plants: APG II. Botanical Journal of the Linnaean Society, v. 141, n. 4, p. 399-436, 2003. Disponível em: <http://onlinelibrary.wiley.com/ doi/10.1046/j.1095-8339.2003.t01-1-00158.x/abstract>. Acesso em: 13 mai. 2013

BERTONI, J. E. A.; MARTINS, F R. Composição florística de uma floresta ripária na Reserva Estadual de Porto Ferreira, SP. Acta Botânica Brasílica, Feira de Santana, v. 1, n. 1, p. 17-26, 1987. Disponível em: <http://www.scielo.br/scielo.php?pid=S010233061987000100003\&script=sci_arttext>. Acesso em: 13 mai. 2013.

BRAGA, A.J.T.; BORGES, E.E.L.L.; MARTINS, S.V. Florística e estrutura da comunidade arbórea de uma floresta estacional semidecidual secundária em Viçosa, MG. Revista Árvore, Viçosa, v. 35, n. 3, p. 493-503, 2011.

BRAZ, D. M.; CARVALHO-OKANO, R. M.; KAMEYAMA, C. Acanthaceae da Reserva Florestal Mata do Paraíso, Viçosa, Minas Gerais. Revista Brasileira de Botânica, São Paulo, v. 25, n. 4, p. 495504, 2002. Disponível em: <http://www.scielo.br/pdf/rbb/v25n4/a13v25n4.pdf>. Acesso em: 13 mai. 2013

CASTRO, P. S.; VALENTE, O.F; COELHO, D.T.; RAMALHO, R.S. Interceptação da chuva por mata natural secundária na região de Viçosa, MG. Revista Árvore, Viçosa, MG, v. 7, p. 76-89, 1983.

CONNEL, J. H.; SLATYER, R. O. Mechanisms of sucession in natural communities and their role in community stability and organization. The American Naturalist, v. 111, n 982, p. 1119-1144, 1977.

ESPÍRITO SANTO, F. D. B.; OLIVEIRA FILHO, A. T.; MACHADO, E. L. M.; SOUZA, J. S.; FONTES, M. A. L.; MARQUES, J. J. G. S. M. Variáveis ambientais e a distribuição de espécies arbóreas em um remanescente de Floresta Estacional Semidecídua Montana no campus da Universidade Federal de Lavras, MG. Acta Botânica Brasílica, São Paulo, v. 16, n. 3, p. 331-356, 2002. Disponível em: <http://www.scielo.br/pdf/abb/v16n3/15396.pdf>. Acesso em: 13 mai. 2013.

FERREIRA JUNIOR, W. G.; SILVA, A. F.; MEIRA NETO, J. A. A.; SCHAEFER, C. E. G. R.; DIAS, A. S.; IGNÁCIO, M.; MEDEIROS, M. C. M. P. Composição florística da vegetação arbórea de um trecho de floresta estacional semidecídua em Viçosa, Minas Gerais, e espécies de maior ocorrência na região. Revista Árvore, Viçosa, MG, v. 31, n. 6, p. 1121-1130, 2007. Disponível em: < http:// www.scielo.br/scielo.php?pid=s0100-67622007000600018\&script=sci_arttext $>$. Acesso em: 14 mai. 2013

GANDOLFI, S.; LEITÃO-FILHO, H. F.; BEZERRA, C. L. F. Levantamento florístico e caráter das espécies arbustivo-arbóreas de uma Floresta Mesófila Semidecídua no município de Guarulhos, SP. Revista Brasileira de Biologia, São Carlos, v. 55, n. 4, p. 753-767, 1995. Disponível em: < http:// www.lcb.esalq.usp.br/publications/articles/1995/1995rbbv55n4p753-767.pdf > . Acesso em: 14 mai. 2013

LEITÃO-FILHO, H. F. Aspectos taxonômicos das florestas do estado de São Paulo. Silvicultura em São Paulo, São Paulo, v. 16, p. 197-206, 1982.

LOPES, W. P.; PAULA, A.; SEVILHA, A. C.; SILVA, A. F. Composição da flora arbórea de um trecho de floresta estacional no Jardim Botânico da Universidade Federal de Viçosa (face sudoeste), Viçosa, Minas Gerais. Revista Árvore, Viçosa, v. 26, n. 3, p. 339-347, 2002. Disponível em: 
<http://www.scielo.br/scielo.php?pid=S0100-67622002000300009\&script=sci_arttext>. Acesso em: 14 mai. 2013

MARANGON, L. C.; SOARES; J. J.; FELICIANO, A. L. P. Florística arbórea da Mata da Pedreira, município de Viçosa, Minas Gerais. Revista Árvore, Viçosa, v. 27, n. 2, p. 207215, 2003. Disponível em: <http://www.scielo.br/scielo.php?script=sci_arttext\&pid $=$ S0100-67622003000200010 > . Acesso em: 14 mai. 2013

MARTINS, S. V. Aspectos da dinâmica de clareiras em uma Floresta Estacional Semidecidual no município de Campinas, SP. 1999. 233f. Tese (Doutorado em Ecologia). Universidade Estadual de Campinas, Campinas.

MARTINS, S. V.; COUTINHO, M. P.; MARANGON, L. C. Composição florística e estrutura de uma floresta secundária no município de Cruzeiro-SP. Revista Árvore, Viçosa, v. 26, n. 1, p. 35-41, 2002.

MARTINS, S. V.; COLLETTI JUNIOR, R.; RODRIGUES, R.R.; GANDOLFI, S. Colonization of gaps produced by death of bamboo clumps in a Semideciduous Mesophytic Forest in south-earstern Brazil. Plant Ecology, [s. I.], v. 172, p. 121-131, 2004. Disponível em: <http://www.jstor.org/ stable/20146593 > . Acesso em: 14 mai. 2013

MENDONÇA, M. P.; LINS, L. V. Lista vermelha das espécies ameaçadas de extinção da flora do estado de Minas Gerais. Belo Horizonte: Fundação Biodiversitas, 2000.

MUELLER DOMBOIS, D.; ELLENBERG, H. Aims and methods in vegetation ecology. New York: Wiley \& Sons, 1974.

OLIVEIRA FILHO, A. T.; ALMEIDA, R. J.; MELLO, J. M.; GAVILANES, M. L. Estrutura fitossociológica e variáveis ambientais em um trecho de mata ciliar do córrego Vilas Boas, Reserva Biológica do Poço Bonito, Lavras (MG). Revista Brasileira de Botânica, São Paulo, v. 17, n. 1, p. 67-85, 1994. Disponível em: <http://www.icb.ufmg.br/treeatlan/Downloads/a22.pdf>. Acesso em 14 mai. 2013

PAULA, A.; SILVA, A. F.; SOUZA, A. L.; SANTOS, F. A. M. Alterações florísticas ocorridas num período de quatorze anos na vegetação arbórea de uma Floresta Estacional Semidecidual em Viçosa-MG. Revista Árvore, Viçosa, MG, v. 26, n. 6, p. 743-749, 2002. Disponível em: <http:// www.scielo.br/pdf/rarv/v26n6/a10v26n6.pdf>. Acesso em 14 mai. 2013.

PEIXOTO, G. L.; MARTINS, S. V.; SILVA, A. F.; SILVA, E. Composição florística do componente arbóreo de um trecho de Floresta Atlântica na Área de Proteção Ambiental da Serra da Capoeira Grande, Rio de Janeiro, RJ, Brasil. Acta Botânica Brasílica, Feira de Santana, v. 18, n. 1, p. 151 160, 2004.

PIELOU, E. C. Ecological diversity. New York: John Wiley \& Sons. 1975.

RIBAS, R. F.; MEIRA NETO, J. A. A.; SILVA, A. F.; SOUZA, A. L. Composição florística de dois trechos em diferentes etapas serais de uma Floresta Estacional Semidecidual em Viçosa, Minas Gerais. Revista Árvore, Viçosa, v. 27, n. 6, p. 821-830, 2003. Disponível em: <http://www.scielo. br/pdf/rarv/v27n6/a08v27n6.pdf>. Acesso em: 14 mai. 2013 
RODRIGUES, L. A.; CARVALHO, D. A.; OLIVEIRA FILHO, A. T.; BOTREL, R. T.; SILVA, E. A. Florística e estrutura da comunidade arbórea de um fragmento florestal em Luminárias, MG. Acta

Botânica Brasílica, São Paulo, v. 17, n. 1, p. 71-87, 2003. Disponível em: <http://www.scielo.br/ scielo.php?script=sci_arttext\&pid=S0102-33062003000100006 > . Acesso em 14 mai. 2013.

RODRIGUES, R. R,; MARTINS, S. V.; BARROS, L. C. Tropical rain Forest regeneration in an area degraded by mining in Mato Grosso State, Brazil. Forest Ecology and Management, $v$. 190, p. 323-333, 2004. Disponível em: <http://www.sciencedirect.com/science/article/pii/ S0378112703005073>. Acesso em 14 mai. 2013.

SHEPHERD, G. J. FITOPAC 1: Manual do usuário. Campinas: UNICAMP. 1996.

SILVA, A. F.; FONTES, N. R. L.; LEITÃO FILHO, H. F. Composição florística e estrutura horizontal do estrato arbóreo de um trecho da Mata da Biologia da Universidade Federal de Viçosa - Zona da Mata de Minas Gerais. Revista Árvore, Viçosa, v. 24, n. 4, p. 397-405, 2000.

SILVA, A. F.; OLIVEIRA, R. V.; SANTOS, N. R. L.; PAULA, A. Composição florística e grupos ecológicos das espécies de um trecho de Floresta Semidecídua Submontana da Fazenda São Geraldo, Viçosa-MG. Revista Árvore, Viçosa, v. 27, n. 3, p. 311-319, 2003. Disponível em: $<$ http://www.scielo.br/scielo.php?pid=S0100-67622003000300006\&script=sci_arttext>. Acesso em: 14 mai. 2013

SILVA, N. R. S.; MARTINS, S. V.; MEIRA NETO, J. A. A.; SOUZA, A. L. Composição florística e estrutura de uma Floresta Estacional Semidecidual Montana em Viçosa, MG. Revista Árvore, Viçosa, MG, v. 28, n. 3, p. 397-405, 2004. Disponível em: <http://www.scielo.br/pdf/rarv/ v28n3/21607.pdf>. Acesso em: 14 mai. 2013.

SILVA JÚNIOR, W. M.; MARTINS, S. V.; SILVA, A. F.; MARCO JUNIOR, P. Regeneração natural de espécies arbustivo-arbóreas em dois trechos de Floresta Estacional Semidecidual, Viçosa, MG. Scientia Forestalis, Piracicaba, v. 66, p. 169-179, 2004. Disponível em: <http://www.ipef.br/ publicacoes/scientia/nr66/cap17.pdf>. Acesso em 14 mai. de 2013.

TABARELLI, M.; VILLANI, J. P.; MANTOVANI, W. Estudo comparativo da vegetação de dois trechos de floresta secundária no Núcleo Santa Virgínia, Parque Estadual da Serra do Mar, SP. Revista do Instituto Florestal, São Paulo, v. 6, p. 1-11, 1994.

TURNER, I. M.; WONG, Y. K.; CHEY, P. T.; IBRAHIM, A. Tree species richness in primary and old secondary tropical forest in Singapore. Biodiversity and Conservation, [s. I.], v. 6, p. 537-543, 1997. Disponível em: <http://link.springer.com/article/10.1023\%2FA\%3A1018381111842\#pa ge-1>. Acesso em: 14 mai. 2013.

VELOSO, H. P.; RANGEL FILHO, A. L. R.; LIMA, J. C. A. Classificação da vegetação brasileira, adaptada a um sistema universal. Rio de Janeiro: Instituto Brasileiro de Geografia e Estatística, 1991. 123p.

\section{Histórico editorial}

Recebido: 15/10/2012

Avaliação e copidesque: 26/10/2012 a 12/03/2013 


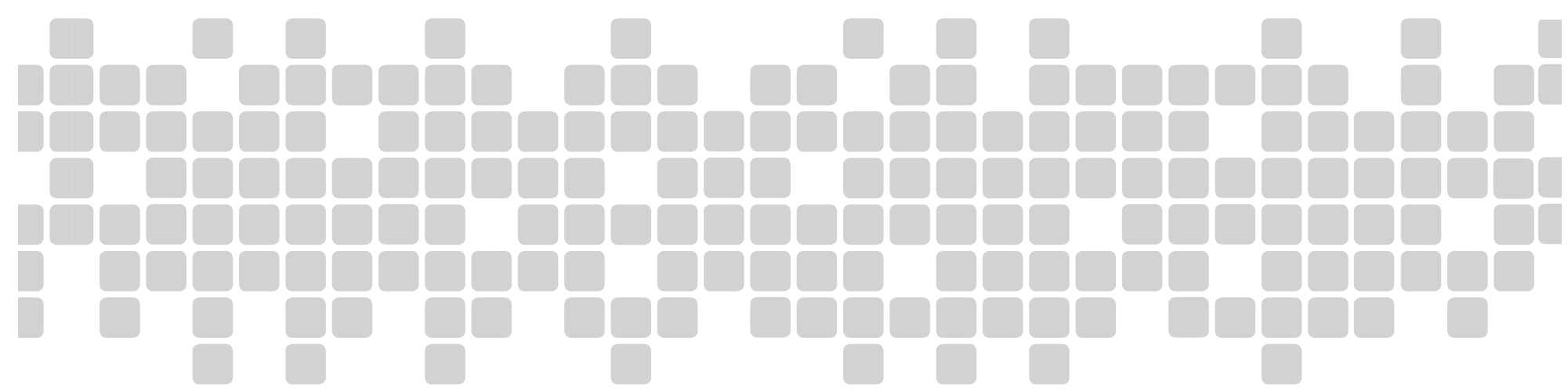

